\title{
Perfil dos usuários atendidos pela fisioterapia dermatofuncional em uma clínica escola de Belém, Pará
}

\author{
Profile of users attended by dermatofunctional \\ physiotherapy in a clinical school of Belém, Pará
}

\author{
Rainara dos Santos Monteiro 1 (1) \\ Ketiane Moura Bezerra2 ${ }^{2}$ (1) \\ Thiffany da Costa Lima 3 (1) \\ Sidney de Assis da Serra Braga 4 (1)
}

\author{
${ }^{1}$ Autora para correspondência. Centro Universitário do Estado do Pará (Belém). Pará, Brasil. rainarasantos3@gmail.com \\ ${ }^{2-4}$ Centro Universitário do Estado do Pará (Belém). Pará, Brasil. keti.ane@hotmail.com, thiffany220@gmail.com, sidneyserrabraga@gmail.com
}

\begin{abstract}
RESUMO | INTRODUÇÃO: A fisioterapia dermatofuncional atua nas disfunções físico-estético-funcionais decorrentes de patologias, procedimentos cirúrgicos e/ou sequelas que afetem direta e indiretamente a integridade do sistema tegumentar. OBJETIVO: Analisar o perfil clínico dos pacientes atendidos em uma Clínica Escola de Fisioterapia de Belém/PA que procuram o serviço de fisioterapia dermatofuncional. MÉTODOS: Esse estudo trata-se de uma análise descritiva, quantitativa e retrospectiva, realizado através da análise prontuários dos pacientes da área de fisioterapia em dermatofuncional (CAAE 25678819.5.0000.5169). RESULTADOS: Foram avaliados 140 prontuários, sendo a maioria dos pacientes do sexo feminino, casados e com idade de média de 36,77 anos e sedentários. Em relação à função psicológica, os pacientes declaravam sentir-se deprimidos e estavam com a autoestima diminuída; a principal queixa mais encontrada foi a adiposidade localizada e o recurso mais utilizado nos atendimentos foi o ultrassom terapêutico de $3 \mathrm{MHz}$. CONCLUSÃO: Através do presente estudo foi possível descrever que no setor de fisioterapia dermatofuncional da clínica escola, a principal queixa foi estética, a disfunção mais tratada foi adiposidade localizada e o recurso mais utilizado foi UST e nortear quanta as queixas mais prevalentes, visto que, a procura por essa especialidade está crescendo. Dessa forma, faz-se necessário conhecer as características do perfil clínico dos pacientes atendidos na área e as diferentes técnicas utilizadas para o tratamento das disfunções.
\end{abstract}

PALAVRAS-CHAVE: Fisioterapia. Perfil de saúde. Estética.

\begin{abstract}
INTRODUCTION: DermatoFuncional Physiotherapy acts on physical-aesthetic-functional dysfunctions resulting from pathologies, surgical procedures and / or sequelae that directly and indirectly affect the integrity of the integumentary system. OBJECTIVE: To analyze the clinical profile of patients treated at a Clinical School of Physiotherapy in Belém / PA who seek the dermatofunctional physiotherapy service. METHODS: This study is a descriptive, quantitative and retrospective analysis, performed through the analysis of medical records of patients in the area of physiotherapy in dermatofunctional. RESULTS: 140 medical records were evaluated, with the majority of patients being female, married and with a mean age of 36.77 years and sedentary. Regarding the psychological function, the patients declared to feel depres sed and had a low self-esteem; the main complaint most found was localized adiposity and the most used resource in the visits was the $3 \mathrm{MHz}$ therapeutic ultrasound. CONCLUSION: Through the present study it was possible to describe that in the dermatofunctional physiotherapy sector of the school clinic, the main complaint was aesthetics, the the most treated dysfunction was localized adiposity and the most used resource was TUS and to guide the most prevalent complaints, since the demand for this specialty is growing. Thus, it is necessary to know the characteristics of the clinical profile of patients seen in the area and the different techniques used to treat dysfunctions.
\end{abstract}

KEYWORDS: Physical Therapy. Health profile. Esthetics. 


\section{Introdução}

A fisioterapia dermatofuncional a cada dia vem ocupando seu espaço e conquistando mais adeptos, os primeiros relatos dos fisioterapeutas trabalhando na área da estética foram em meados dos anos 70, porém apenas nos anos 90 surgiram as primeiras publicações nacionais de cunho científico' ${ }^{1}$. No Brasil essa especialidade foi reconhecida pelo COFFITO através da resolução $n^{\circ} 362$, de 20 de maio de 2009 e tornouse mais popular no país em virtude da procura por tratamentos que objetivavam, além da recuperação física, a melhora da saúde, bem-estar e autoestima ${ }^{2,3}$.

O fisioterapeuta que trabalha nesta área tem como dever manter e promover a ótima função física, visando também o bem-estar e a qualidade de vida dos pacientes ${ }^{4}$. Abordando as disfunções físico-estético-funcionais decorrentes de patologias, procedimentos cirúrgicos e/ou sequelas que afetem direta e indiretamente a integridade do sistema tegumentar ${ }^{5}$. Dessa forma, esta especialidade é de extrema importância dentro da fisioterapia, visto que sua atuação não se restringe somente a estética, mas é de suma relevância na parte reparadora.

A atuação da fisioterapia dermatofuncional é bem ampla e variada, tanto no aspecto preventivo como de reabilitação, dentro das seguintes subáreas: disfunções dermatológicas, como dermatoses, acne, rosácea; disfunções vasculares, como fleboedemas, lipedemas, linfedemas; disfunções da cicatrização, como queimaduras, cicatrizes hipertróficas, queloides, deiscências, aderências, úlceras; disfunções estéticas, como fibroedema geloide (FEG), estrias atróficas, lipodistrofias; e pós-operatórios de cirurgias plásticas reparadoras com ou sem linfadenectomias, de cirurgias plásticas estéticas e de cirurgias vasculares, dentre outras ${ }^{6}$. Além de que no Guide to physical therapist practice, publicado pela Associação NorteAmericana de Fisioterapia (APTA), foi esclarecido que a fisioterapia dermatofuncional é uma área referida como a responsável pela manutenção do sistema tegumentar como um todo?.

Anteriormente os tratamentos estéticos em geral eram tratados como empíricos, a fisioterapia dermatofuncional trouxe a área, maior especialidade e cientificidade ${ }^{4}$ o que é ressaltado por Tacani ${ }^{5}$ ao proferir que os recursos fisioterapêuticos, quando utilizados de forma coerente e fundamentada no modelo científico atual, podem melhorar várias disfunções do sistema tegumentar. No levantamento bibliográfico realizado por Milani ${ }^{3}$, conclui-se que há na literatura científica embasamento para justificar a escolha dos diversos recursos utilizados nas patologias englobadas por essa área da fisioterapia. Segundo os autores há necessidade de se ampliar o número de pesquisas científicas, consolidando assim a fisioterapia dermatofuncional como uma área relevante no contexto da saúde brasileira.

Conhecer o perfil dos pacientes atendidos no setor da fisioterapia dermatofuncional é fundamental para os acadêmicos do curso e profissionais, pois permitirá um melhor planejamento na elaboração de condutas de acordo com as características e necessidades dos pacientes. Diante do exposto, o presente estudo teve como objetivo analisar o perfil clínico dos pacientes atendidos em uma Clínica Escola de Fisioterapia de Belém/PA que procuram o serviço de fisioterapia dermatofuncional.

\section{Metodologia}

Trata-se de uma análise descritiva, quantitativa e retrospectiva. Foram analisados os prontuários dos pacientes atendidos no setor de Fisioterapia em Dermatofuncional de uma clínica escola de Belém do Pará, no período de agosto de 2017 a setembro de 2019. Foram incluídos os dados que constavam nos portuários dos pacientes atendidos e excluídos os prontuários que apresentavam dados incompletos.

A coleta de dados foi realizada por meio da ficha de avaliação disponibilizada através do prontuário eletrônico Fisio Office que é o sistema disponível na clínica escola de Fisioterapia, que contém informações sobre a ficha de avaliação e a evolução de cada paciente. Foram coletadas variáveis como: idade, sexo, estado civil e cor de pele, principal motivo da procura (principais queixas), os hábitos de vida, a função psicológica e os recursos fisioterapêutico utilizados nos atendimentos. Este estudo foi aprovado pelo Comitê de Ética em Pesquisa do Centro Universitário do Estado do Pará (CAAE 25678819.5.0000.5169). Os dados obtidos foram armazenados em uma planilha do programa Microsoft Excel, versão 2013 e posteriormente realizou-se a análise estatística descritiva no programa Epi InfoTM, sendo representados cálculo de porcentagens, medidas de tendência central (médias) e medidas de dispersão (desvio padrão). 


\section{Resultados}

Foram avaliados 155 prontuários dos pacientes em tratamento fisioterapêutico, 15 destes prontuários foram excluídos por dados incompletos, sendo então utilizados 140 prontuários restantes que expressar as seguintes características sociodemográficas que estão apresentadas na Tabela 1, onde são elencadas as informações ligadas ao sexo, a idade, estado civil e cor da pele. Que caracterizaram o perfil sociodemográfico com média de idade de 36, 77 (DP $\pm 12,14$ ) anos, onde $113(80,7 \%)$ eram do sexo feminino e $27(19,2 \%)$ do sexo masculino. Quanto ao estado civil, eram solteiros 35 (25\%), casados 77 (55\%), divorciados 19 (13,5\%) e viúvos 9 (6,4\%), com relação a cor da pele $71(50,7 \%)$ eram pardos.

Tabela 1. Registro das características sociodemográfico

\begin{tabular}{ccc}
\hline Variáveis & Média (Desvio padrão) & $\mathbf{N}(\%)$ \\
\hline Idade (anos) & $36,77 \pm 12,14$ & \\
Sexo & & $113(80,7 \%)$ \\
Feminino & - & $27(19,2 \%)$ \\
Masculino & \\
& & \\
& & $35(25 \%)$ \\
Estado civil & $77(55 \%)$ \\
Solteiro(a) & $19(13,5 \%)$ \\
Casado(a) & $9(6,4 \%)$ \\
Viúvo(a) & \\
Divorciado(a)/separado(a) & \\
Cor da pele: & \\
Branca & \\
Parda & $46(32,8 \%)$ \\
Negra & $71(50,7 \%)$ \\
Amarela & $16(11,4 \%)$ \\
& $7(5 \%)$ \\
\hline
\end{tabular}

Fonte: Dados da pesquisa, ano 2019.

Em relação aos hábitos de vida foi possível observar que dos prontuários analisados eram sedentários 89 (61,2\%), não etilistas 91 (65\%) e não tabagistas 109 (77,8\%). Desses pacientes, faziam uso de medicamentos 78 (55,7\%) (Tabela 2).

Tabela 2. Registro dos hábitos de vida

\begin{tabular}{ccc}
\hline Variáveis & $\mathbf{N}^{\circ}$ & $(\%)$ \\
\hline Atividade física & & $36,4 \%$ \\
Sim & 51 & $61,2 \%$ \\
Não & 89 & $35 \%$ \\
Alcoolismo & & $65 \%$ \\
Sim & 49 & $22,1 \%$ \\
Não & 91 & $77,8 \%$ \\
Tabagismo & 31 & \\
Sim & 109 & $55,7 \%$ \\
Não & & $44,2 \%$ \\
Medicamentos & 78 & \\
Sim & 62 & \\
Não & &
\end{tabular}

Fonte: Dados da pesquisa, ano 2019 
Em relação a função psicológica dos pacientes que procuraram atendimento fisioterapêutico dermatofuncional, foi constatado ao analisar as variáveis "Como se sente" que $52(37,1 \%)$ relataram sentir-se entristecido e quanto a "Autoestima" $65(46,4 \%)$ com autoestima diminuída. Ao serem questionados se tinham alguma atividade limitada por conta de sua patologia responderam não 96 (68,5\%) (Tabela 3).

Tabela 3. Função psicológica

\begin{tabular}{ccc}
\hline Variáveis & Média (Desvio padrão) & $\mathbf{N}(\%)$ \\
\hline Função psicológica & - & \\
(Como se sente) & & $21(15 \%)$ \\
Sente-se alegre & & $41(29,2 \%)$ \\
Sente-se ansioso & $52(37,1 \%)$ \\
Sente-se entristecido & $26(18,5 \%)$ \\
Sente-se deprimido & - & \\
Função psicológica (Autoestima) & & \\
Autoestima aumentada & - & $23(16,4 \%)$ \\
Autoestima diminuída & & $65(46,4 \%)$ \\
Autoestima normal & $43(30,7 \%)$ \\
Atividades limitadas & & \\
Sim & & $44(31.4 \%)$ \\
Não & $96(68,5 \%)$ \\
\hline
\end{tabular}

Fonte: Dados da pesquisa, ano 2019.

Dentre as principais disfunções encontradas e descritas nos prontuários constam os dados referentes às queixas principais e tratamentos fisioterapêuticos propostos na área de fisioterapia dermatofuncional. Foram encontrados sete tipos de disfunções: Adiposidade localizada, rugas, FEG, úlcera, flacidez, estrias e acne. Sobre as queixas principais, observaram- se os seguintes resultados: adiposidade localizada foi o maior motivo de procura ao serviço com 51 (37,10\%), seguida de úlcera 31 (22,80\%), acne 29 (21,40\%), flacidez 26 (19,20\%), FEG 24 (17,80\%), estrias 16 $(12,10 \%)$, rugas 14 (10\%). Observa-se também que 51 (37,10\%) dos pacientes que procuram o serviço de fisioterapia por queixa de adiposidade localizada, contudo $89(61,2 \%)$ não realizam nem um tipo de atividade física, (Figura 1).

Figura 1. Gráfico referente a análise do principal motivo do tratamento Dermatofuncional

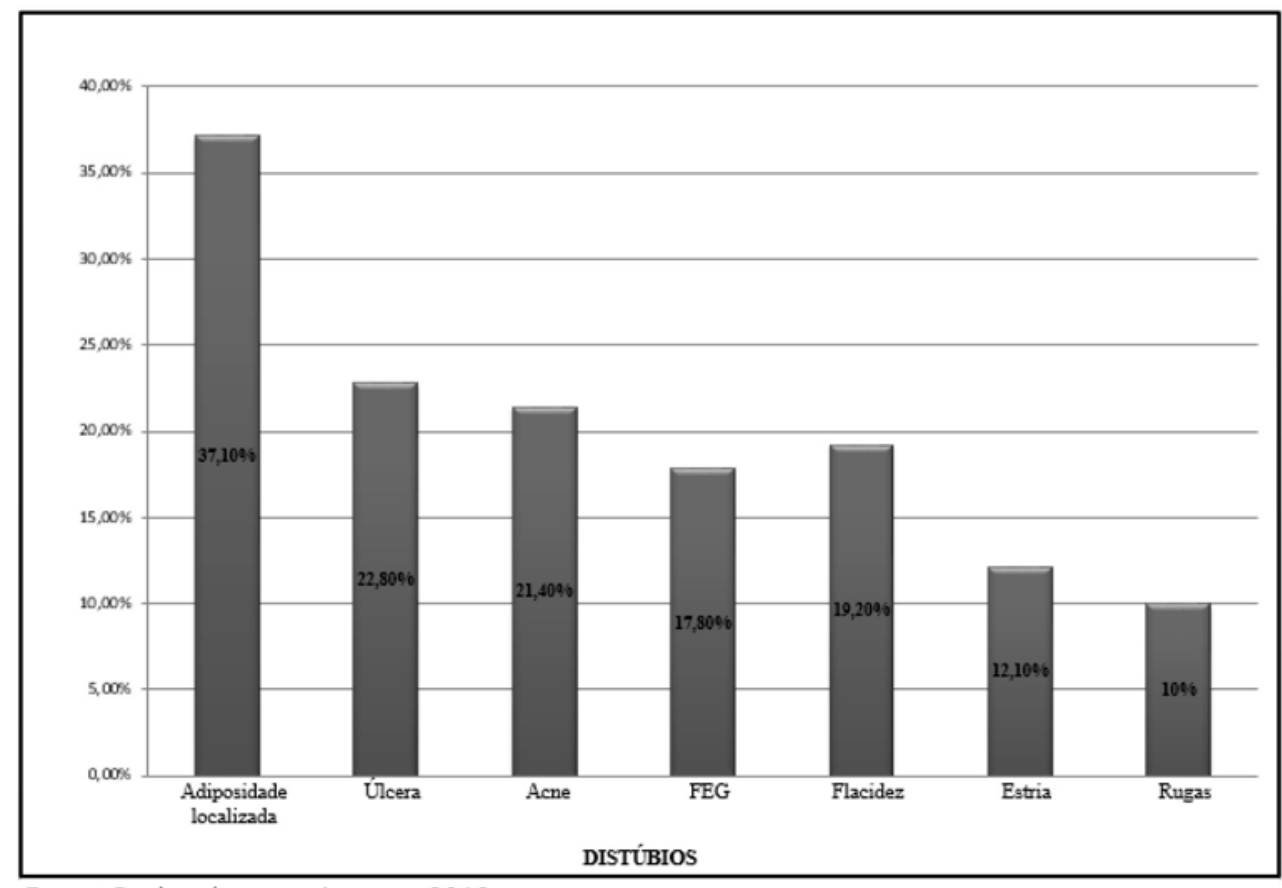

Fonte: Dados da pesquisa, ano 2019. 
$\mathrm{Na}$ figura 2, apresentam-se as características dos atendimentos procurados e realizados pelos pacientes. Quanto ao motivo que os levaram a buscar pelo serviço de fisioterapia dermatofuncional, observou-se que a maiorias dos pacientes referiram queixa estética. Deste modo no que se refere ao tipo de recursos realizados com maior frequência, notou-se que o mais utilizado nos atendimentos foi o ultrassom terapêutico (UST) 98 (70\%), podendo ser utilizado isolado ou associado a mais procedimentos. Observou-se ainda que o recurso endermologia foi utilizado por 66 (47,80\%) dos pacientes, seguido por: argiloterapia 63 (45,70\%), corrente russa 45 (33,50\%), massagem modeladora 45 (32,80\%), microcorrente $44(32,10 \%)$, drenagem linfática $42(30 \%)$, peeling de diamante $35(25,70 \%)$, radiofrequência 32 (23,50\%), LASER 31 (22,80\%), alta frequência 29 (21,40\%).

Figura 2. Gráfico referente à análise dos recursos mais utilizado

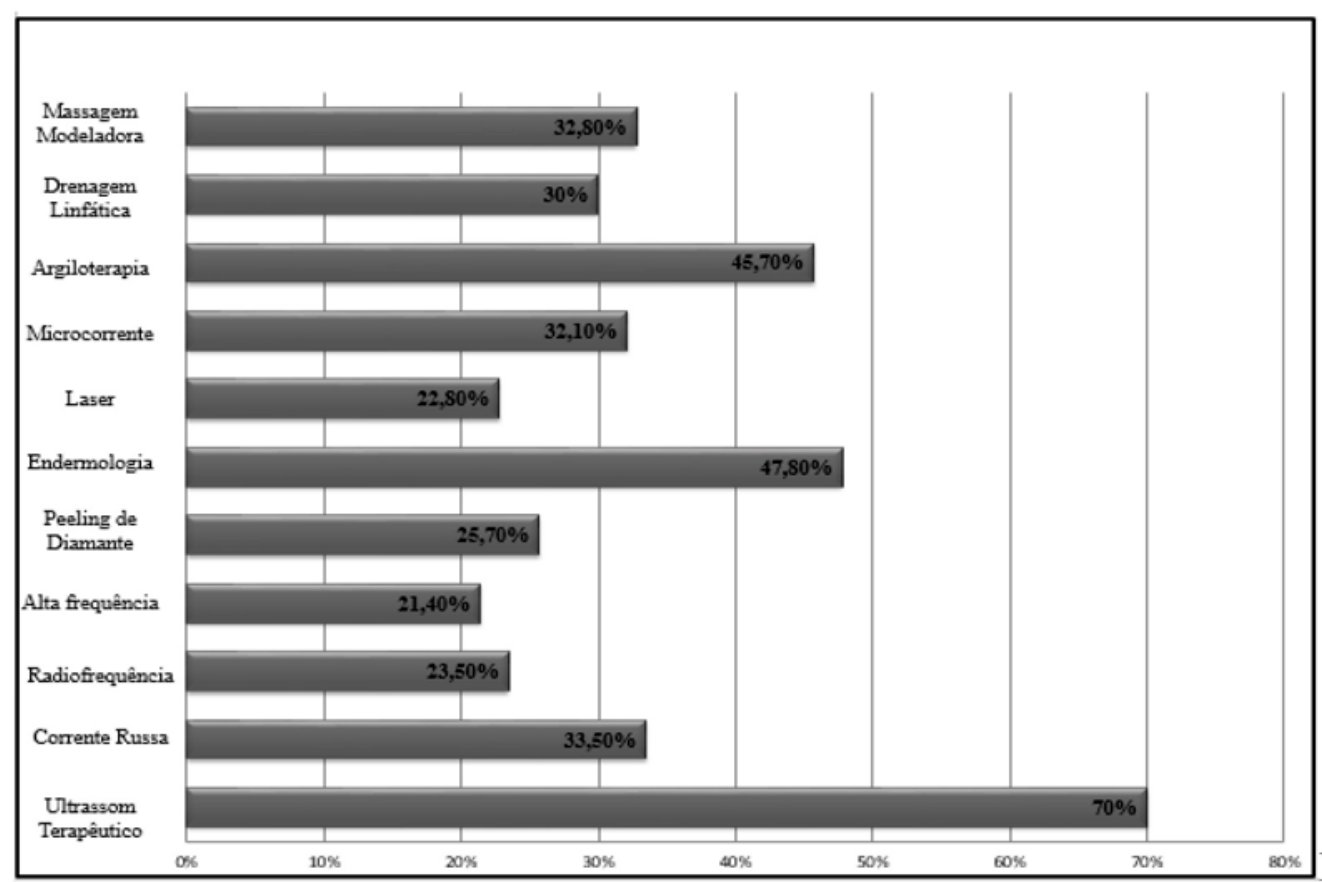

Fonte: Dados da pesquisa, ano 2019.

\section{Discussão}

Ao analisar os dados coletados nos prontuários clínicos, observou-se que a prevalência dos pacientes atendidos foi do sexo feminino $80,7 \%$, apesar do serviço ser oferecido para ambos os sexos, essa prevalência pode ser resultante do padrão de beleza imposto pela sociedade, no qual prevalece o padrão magro, esbelto e sem nenhuma gordura somado com a insatisfação da imagem corporal8,9,10.

O principal motivo que levou os pacientes a procurarem o serviço de fisioterapia dermatofuncional foi referente à queixa estética, sendo a adiposidade localizada 37,10\% o maior motivo de procura ao serviço. Corroborando com os achados de Torres et al. ${ }^{4}$ que em sua pesquisa apresentou que $47,50 \%$ dos entrevistados referiram queixa estética sendo $43,75 \%$ dessas queixas indicavam mais de uma insatisfação e 33,7\% dessa insatisfação estava relacionada a adiposidade localizada. A flacidez da pele, o excesso de gordura corporal e o FEG causam problemas funcionais e emocionais, levando a diminuição da autoestima podendo estar interferindo nas relações interpessoais impactando na qualidade de vida das mulheres ${ }^{11}$.

Nesta pesquisa, $37,10 \%$ dos pacientes buscaram o serviço com queixa principal de adiposidade localizada em contrapartida $61,2 \%$ não realizavam atividade física. A atividade física é um fator importante para manutenção e perda de peso corporal, tendo como benefícios o controle da taxa metabólica, melhora na qualidade do sono, redução dos níveis de ansiedade e estresse, além disso, os exercícios físicos juntamente com as dietas hipocalóricas promovem a diminuição da gordura corporal e o aumento da massa magra, enquanto o sedentarismo é um importante fator de risco para a obesidade $12,13,14,15$. 
Durante a análise dos dados referente a função psicológica notou-se que os pacientes que procuraram atendimento relataram sentir-se entristecido e com autoestima diminuída, corroborando parcialmente com o estudo de Skopinski et al. ${ }^{16}$ realizado com mulheres no período da pós-menopausa atendidas no serviço de fisioterapia dermatofuncional, no qual, a maior parte da amostra demonstrou insatisfação com a imagem corporal e apresentaram sintomas sugestivos de depressão.

O tratamento de úlcera foi a segunda queixa mais procurada, no setor de fisioterapia dermatofuncional da clínica, demostrando uma característica do perfil dos pacientes que são atendidos na clínica escola nessa especialidade. $O$ resultado do presente estudo difere de outras pesquisas onde a principal procura pelo tratamento da fisioterapia dermatofuncional estava relacionada com linfedema $a^{6,17}$.

Vários estudos têm demonstrado a vulnerabilidade masculina às enfermidades crônicas e graves, observando-se que os homens são mais suscetíveis à doença crônica mal controlada e a causas externas como traumas decorrentes de acidentes ${ }^{18}$. Não obstante Rogenski e Santos ${ }^{19}$ observaram em seu estudo realizado no Hospital Universitário da USP uma dominância do sexo masculino tanto para o grupo com e sem úlcera 52,4 e 56,6\%, respectivamente. Resultado igual ao obtido na presente pesquisa, no qual, houve maior prevalência no sexo masculino para queixa se úlcera e o autor observou também que a idade dos pacientes com úlcera variou de 22 a 95 anos.

Dentre os recursos disponibilizados a fisioterapia dermatofuncional, o ultrassom terapêutico de de $3,0 \mathrm{MHz}$ destaca-se neste estudo, como o recurso mais utilizado na adiposidade localizada. No estudo de Texeira et al.20 realizado com 27 mulheres sedentárias observou-se que a utilização do ultrassom $3 \mathrm{MHz}$, no modo contínuo, apresentou resultados na redução da adiposidade da região infra-abdominal, quando associado a ativos lipolíticos ou usado de forma isolada.

O tratamento com ultrassom de $3 \mathrm{MHz}$ é indicado para tecidos superficiais enquanto que o ultrassom de $1 \mathrm{MHz}$ é indicado para tecidos profundos, podendo ser associado com a fonoforese, introdução de princípios ativos através da pele utilizando o ultrassom ${ }^{21,22}$.
Dentre seus efeitos fisiológicos destaca-se a vasodilatação, aumento da permeabilidade da membrana, neovascularização, aumento do metabolismo e aumento da extensibilidade das fibras de colágeno ${ }^{21}$.

O segundo recurso mais utilizado foi a endermoterapia que consiste em uma técnica terapêutica que se utiliza de um aparelho que permite uma dupla ação sinérgica de aspiração e mobilização dérmica, onde é utilizada a pressão negativa na sucção, associada ao rolamento exercido pelos rolos presentes no cabeçote, além disso, tem como efeitos fisiológicos a vasodilatação, melhora de oxigenação e nutrição tecidual, melhora do sistema linfático e auxilia na eliminação de toxinas ${ }^{23}$. Neste estudo notou-se que $47,80 \%$ dos terapeutas usaram a endermoterapia, justamente por se tratar de um recurso que contribui no tratamento da adiposidade localizada, atuando no tecido adiposo, estruturas vasculares e linfáticas estimulando e transformando a gordura em glicerol para que seja absorvida pela circulação e eliminada pelo organismo, além de promover a remodelação das células adiposas e melhora do contorno corporal ${ }^{3,24}$.

Filippo e Júnior ${ }^{25}$ em seu estudo clínico avaliaram a segurança e eficácia de técnica que combinam quatro diferentes tecnologias: o ultrassom, radiofrequência, Light Emitting Diodes (LEDs) e endermologia, em 21 mulheres com idades entre 24 e 39 anos, foi possível notar redução de $2,85 \mathrm{~cm}$ na medida da circunferência abdominal e uma média 6,83 no grau da escala de avaliação de satisfação com o tratamento, sendo a endermologia uma técnica bem empregada em pacientes com queixa de adiposidade localizada podendo ser ainda associada ao ultrassom.

O terceiro recurso mais utilizado foi a argiloterapia e de acordo com o estudo de Toyoki e Oliveira ${ }^{26}$ este é um tratamento que possui inúmeros benefícios à saúde e à estética da pele como a melhora da circulação sanguínea promovendo a remoção de toxinas, a tonificação, nutrição da pele, além de apresentarem propriedades antissépticas, cicatrizantes, anti-inflamatórias, esfoliantes/hidratantes, ressalta-se ainda que cada argila possui sua particularidade no que diz respeito aos fins terapêuticos. Existem vários tipos de argila: branca, amarela, vermelha, rosa, preta e verde, isso deve-se aos diversos minerais em sua composição como: alumínio, ferro, magnésio, silício e entre outros que são os responsáveis por sua coloração e cada uma destas substâncias dará ação terapêutica para a argila ${ }^{27}$. 


\section{Conclusão}

Através do presente estudo foi possível descrever que no setor de fisioterapia dermatofuncional da clínica escola, a principal queixa foi estética, a disfunção mais tratada foi adiposidade localizada e o recurso mais utilizado foi UST e nortear quanto as queixas mais prevalentes, visto que, a procura por essa especialidade está crescendo. Dessa forma, faz-se necessário conhecer as características do perfil clínico dos pacientes atendidos na área e as diferentes técnicas utilizadas para o tratamento das disfunções.

\section{Contribuições dos autores}

Monteiro RS participou da concepção, delineamento, busca e análise estatística dos dados da pesquisa, interpretação dos resultados, redação do artigo científico. Bezerra KM participou da coleta de dados da pesquisa e redação do artigo científico. Lima TC participou da coleta de dados da pesquisa e redação do artigo científico. Braga SAS orientou o estudo e participou da redação. Todos os autores participaram da revisão crítica do manuscrito quanto ao conteúdo.

\section{Conflitos de interesses}

Nenhum conflito financeiro, legal ou político envolvendo terceiros (governo, empresas e fundações privadas, etc.) foi declarado para nenhum aspecto do trabalho submetido (incluindo, mas não se limitando a subvenções e financiamentos, participação em conselho consultivo, desenho de estudo, preparação de manuscrito, análise estatística, etc.).

\section{Referências}

1. Paiva PCB, Castro P, Andrade GM, Coutinho BG. Levantamento do perfil dos pacientes e tratamentos mais utilizados em uma clínica de dermato-funcional em pelotas-rs. Revista Campo do Saber. 2018;4(6):25-30.

2. Conselho Regional de Fisioterapia e Terapia Ocupacional de Rio de Janeiro. Definição Fisioterapia [Internet]. 2018. Disponível em:http://www.crefito2.gov.br/fisioterapia/definicao/--32.html

3. Milani GB, João SMA, Farah EA. Fundamentos da Fisioterapia Dermato-Funcional: Revisão de literatura. Rev Fisioter Pesqui. 2006;13(1):37-43. doi: 10.1590/fpusp.v13i1.76159

4. Torres JS, Ibiapina LC, Ferreira LHM, Moura RO, Carvalho KC. Perfil dos usuários atendidos pela fisioterapia dermato-funcional em Teresina/PI. Fisioter Bras. 2014;15(2):84-88. doi: 10.33233/ fb.v15i2.318
5. Tacani RE, Campos MSMP. A fisioterapia, o profissional fisioterapeuta e seu papel em estética: Perspectivas históricas e atuais. Rev Bras Cienc Saúde 2004;2(4):46-9.

6. Tacani PM, Machado AFP, Tacani RE. Perfil clínico dos pacientes atendidos em fisioterapia dermatofuncional na clínica da universidade municipal de São Caetano do Sul-USCS. Rev Bras Ciên Saúde. 2009;7(21):36-41. doi: 10.13037/rbcs.vol7n21.300

7. Leite CBS, Sousa ML, Zaramella SA, D'afonsêca A. Atuação do fisioterapeuta dermatofuncional e seu reconhecimento pela classe médica. Rev Inspirar Mov Saude. 2013;5(1):1-4.

8. Tacani PM, Tacani RE, Machado AFP, Peroni AE, Silva MA, Freita JOG. Perfil clínico de pacientes atendidos em fisioterapia assistencial à cirurgia plástica: análise retrospectiva. ConScientiae Saúde.2013;12(2):290-297. doi: 10.5585/conssaude.v12n2.4276

9. Tacani PM, Machado AFP, Souza DAA, Tacani RE. Efeito da massagem clássica estética em adiposidades localizadas: estudo piloto. Rev Fisioter Pesqui. 2010;17(4):352-7. doi: 10.1590/S1809$\underline{29502010000400013}$

10. Pinto MCCS, Pereira LP, Bacelar IA. O uso do ultrassom no tratamento de lipodistrofia localizada-revisão de literatura. Revista Saúde em Foco. 2018, (10): 485-495.

11. Silva RMV, Cavalcanti RL, Rego LMF, Nunes PFL, Meyer PF. Efeitos do Kinesio Taping ${ }^{\circledR}$ no tratamento do Fibro Edema Gelóide: Ensaio clínico controlado randomizado e cego. MTP\&RehabJournal. 2014;12: 261-278.

12. Kenney WL, Wilmore JH, Costill DL. Fisiologia do esporte e do exercício. 5.ed. São Paulo: Manole; 2013.

13. Schveitzer V, Claudino R. Importância da atividade física durante o processo de envelhecimento. Efdeports. 2010;14(141):1.

14. Fonseca-Junior SJ, Sá CGAB, Rodrigues PAF, Oliveira AJ, Fernandes-Filho J. Exercício físico e obesidade mórbida: uma revisão sistemática. Arq Bras Cir Dig. 2013; 26(Supl 1):67-73. doi: 10.1590/S0102-67202013000600015

15. Freire RS, Lélis FLO, Fonseca Filho JA, Nepomuceno MO, Silveira MF. Prática regular de atividade física: estudo de base populacional no Norte de Minas Gerais, Brasil. Rev Bras Med Esporte. 2014;20(5):345-9. doi: 10.1590/1517-86922014200502062

16. Skopinski F, Resende TL, Schneider RH. Imagem corporal, humor e qualidade de vida. Rev Bras Geriatr Gerontol. 2015;18(1):95-105. doi: 10.1590/1809-9823.2015.14006

17. Tacani PM, Arcas CS, Silva FFD, Carasco M, Machado AFP, Tacani RE. Perfil dos pacientes atendidos em fisioterapia dermatofuncional. Fisioter Bras.2016;14(5):351-356. doi: 10.33233/fb.v14i5.417 
18. Sousa RG, Oliveira TL, Lima LR, Stival MM. Fatores associados à úlcera por pressão (UPP) em pacientes críticos: revisão integrativa da literatura. Univ Ci Saúde. 2016;14(1):77-84. doi: 10.5102/ucs. v14i1.3602

19. Rogenski NMB, Santos VLCG. Estudo sobre a incidência de úlceras por pressão em um hospital universitário. Rev Latino-am Enfermagem. 2005;13(4):474-80. doi: 10.1590/S010411692005000400003

20. Teixeira CG, Chartuni JN, Sossai LS. Efeitos do ultrassom 3MHZ associado a ativos lipolíticos na adiposidade infraabdominal: Ensaio Clínico Randomizado. Perspect Online Cienc Biol Saúde. 2011;1(1). doi: $10.25242 / 8868112011515$

21. Borges FS. Dermato-Funcional: Modalidades Terapêuticas nas disfunções estéticas. 1.ed. São Paulo: Phorte; 2006.

22. Hoppe S, Marin MF, Simões NDP, Simionato G. Fonoforese na redução da adiposidade abdominal. Rev Bras Terap e Saúde. 2010;1(1):13-26. doi: 10.7436/rbts-2010.01.01.02

23. Costa PS, Mejia DPM. Efeitos fisiológicos da endermoterapia combinados a massagem modeladora no tratamento de gordura localizada na região do abdômen [monografia]. Goiânia: Faculdade Cambury; 2013.

24. Palma MR, Araujo MFS, Nakamura JYM, Silva BB, Najas CS, Pacagnelli FL et al. Ação da Endermologia no Tratamento da Lipodistrofia Localizada. Colloquium Vitae. 2012;4(especial);84-90.

25. Filippo AA, Salomão Júnior A. Tratamento de gordura localizada e lipodistrofia ginóide com terapia combinada: radiofrequência multipolar, LED vermelho, endermologia pneumática e ultrassom cavitacional. Surg Cosmet Dermatol 2012; 4(3):241-6.

26. Toyoki BK, Oliveira ACT. Argiloterapia: levantamento dos constituintes e utilizações dos diferentes tipos de argila [monografia]. São Paulo: Centro Universitário das Faculdades Metropolitanas Unidas; 2015.

27. Abel A. Caracterização de argilas para uso em saúde e estética [monografia]. Criciúma: Universidade do Extremo Sul Catarinense; 2009. 